\title{
Synthetic Monoclonal Antibody Designed for Novel SARS-nCoV-2 Spike-S1 Protein Antigenic Targeted Epitope of Receptor Binding Domain Inhibit to Prevent Viral Entry
}

\section{Zainularifeen Abduljaleel ( $\nabla$ zaabduljaleel@uqu.edu.sa )}

Department of Medical Genetics, Faculty of Medicine, Umm Al-Qura University, Makkah 21955, Kingdom of Saudi Arabia. 2 Science and Technology Unit, Umm Al-Qura University, P.O.Box 715, Makkah 21955, Kingdom of Saudi Arabia. 3 Molecular Diagnostics Unit, Al-Noor Specialized Hospital P.O.Box: 6251, Makkah, Kingdom of Saudi Arabia. 4 Molecular Diagnostics Unit, Department of Molecular Biology, The Regional Laboratory, Ministry of Health (MOH), P.O.Box 6251, Makkah, Saudi Arabia.

\section{Faisal A. Al-Allaf}

Department of Medical Genetics, Faculty of Medicine, Umm Al-Qura University, Makkah 21955, Kingdom of Saudi Arabia. 2 Science and Technology Unit, Umm Al-Qura University, P.O.Box 715, Makkah 21955, Kingdom of Saudi Arabia.

\section{Research Article}

Keywords: COVID19, severe acute respiratory syndrome-coronavirus (SARS-CoV-2), human receptor angiotensin converting enzyme-2 (hACE2), receptor binding domain (RBD), monoclonal antibody, spike (S) protein, cellular immune responses

Posted Date: September 1st, 2020

DOI: https://doi.org/10.21203/rs.3.rs-68933/v1

License: (9) (1) This work is licensed under a Creative Commons Attribution 4.0 International License. Read Full License

Version of Record: A version of this preprint was published at Molecular Diversity on September 1st, 2020. See the published version at https://doi.org/10.1007/s11030-022-10449-x. 


\section{Abstract}

Severe acute respiratory syndrome (SARS) is developing disease caused by novel coronavirus (COVID19). This situation has urgently prompted many pharma and R\&D research companies and public research health sectors to concentrate their efforts on research for effective therapeutics. SARS-nCoV-2 as a spike (S) protein was a targeted for the development of monoclonal antibody and therapeutics for the prevention and treatment. We developed monoclonal antibody by deep mutational scanning to characterize the effects of mutations in an antibody variable fragment on its based on expression levels, specificity, stability, and affinity for antigenic specific epitope to the Spike-S-RBD. Further to make this antibody multipoint core mutations to improve contacts between specific Fv light and heavy chains to the targeted antigen of RBD. This antibody combined enhancing mutations yielding with higher binding affinity and substantially improved stability in between RBD and antibody. Overall, this antibody may well prevent into the cell interaction with RBD-hACE2 to viral entry and prevention. SARS-nCoV-Spike-S monoclonal antibodies potently inhibited SARS-nCoV-2-Spike-S mediated entry into cells, indicating that cross-neutralizing antibodies targeting conserved $S$ epitopes can be elicited based upon vaccination.

\section{Introduction}

The occurrence of coronavirus disease in December 2019 (COVID-19) is a respiratory illness that can spread from person to person. The virus that causes COVID-19 is a novel coronavirus that was first identified during an investigation into an outbreak in Wuhan, China. A newly identified novel coronavirus SARS-CoV-2, formerly known as 2019-nCoV is causing pneumonia-associated respiratory syndrome (Wu et al., 2020). After analysis of genome sequences of SARS-CoV-2 samples obtained from different infected patients, SARS-CoV-2 shares high sequence identity with SARS-CoV (Zhou et al., 2020 and Lu et al., 2020). Compared to SARS-CoV, transmitted from human-to-human of SARS-CoV-2 seems to be greater. As of February 2020, at least 25 countries reported $>70,000$ cases of SARS- CoV-2 infection. A Complication of this virus is a Patients with $\mathrm{nCoV}-2$ have had mild to severe respiratory illness with symptoms of fever, cough and shortness of breath also some patients have pneumonia in both lungs, multi-organ failure and in some cases death. At present people with nCoV-2, disease confirmed by molecular testing by reverse transcription polymerase chain reaction: RT-PCR, weak, late or absent antibody responses have been reported (Zhao J et al., 2019, Okba et al., 2020, Lin et al., 2020). Reports suggest that the majority of patients develop antibody response only in the second week after onset of symptoms. (Liu et al., 2020, Wolfel et al., 2019, Zhou et al., 2020) This means that a diagnosis of nCoV-2 infection based on antibody response will often only be possible in the recovery phase, when many of the opportunities for clinical intervention or interruption of disease transmission have already passed.

Unfortunately, There are currently no specific vaccine or specific antiviral drugs to protect against COVID19. Researchers across the world including several biotech and pharma R\&D companies are investigating preventative approaches and treatment options for $\mathrm{nCoV}-2$, including vaccines, monoclonal antibodies, oligonucleotides, small peptides, interferon type 2 alpha and Beta, and small-molecule drugs (Tang et al., 2008). Since only a way suggests antibody-mediated humoral response is crucial for preventing viral 
infections. A subset of these antibodies, which reduce viral infectivity by binding to the surface epitopes of viral particles and thereby blocking the entry of the virus to an infected cell, are defined as neutralizing antibodies (NAbs) (Varghese et al., 2004).

The CoV-2 spike (S1) protein is a key target for urgently needed vaccines, therapeutic antibodies, and diagnostics (Xintian et al., 2020). Spike proteins are the visible protrusions on the surface of COVID19, which give the virus its characteristic, crown-like appearance (Figure 1). These homotrimeric proteins are heavily glycosylated, with each comprising two distinct subunits (S1 and S2). The role of Spike is to act as a molecular key, which it achieves by recognizing and binding to specific hACE2 cell-surface receptors present on the surface of cells, through the $\mathrm{S} 1$ receptor-binding domain. When S1 binds to hACE2, Spike undergoes dramatic structural changes to alter the conformation of hACE2 and mediate entry of the virus into the cell. However, as Spike proteins must project into the external environment to effectively bind cellsurface receptors, they are exposed to recognition by the immune system. This makes Spike the immunodominant coronavirus antigen, causing it to elicit a strong neutralizing antibody response $(\mathrm{Ng}$ et al., 2016) that has made it the focal target of diagnostic and vaccine development.

In the method of in-silico design allows for rapid iteration around potential solutions without the unique variables that traditional in vivo subjects may possess. Traditional in vivo methods test for the likelihood of producing an antibody that triggers the body's immune system to fight against pathogens. This techniques can be developed and delivered remotely, and do not require the on-site presence needed for traditional wet labs.

The aim of this study was to describe to construct design of synthetics peptide monoclonal antibody (mAbs) for Spike S1 protein to target of specific antigenic epitopes present in RBD. The key residues between RBD and monoclonal antibody can be particularly identified to provide important implications for the vaccines against $\mathrm{nCoV}-2$. Because spike protein is main antigenic component that is responsible for inducing host immune responses, neutralizing antibodies and protective immunity against virus infection. Spike protein has therefore been selected as an important target for coronavirus vaccine and anti-viral development. Therefore, key residues of interface between an monoclonal antibody and the RBD antigen can be optimized to generate with high affinity.

\section{Materials And Methods}

\section{Dataset preparation and selection}

Numbers of antibody structures was currently around more than 2,000 depositions are available in NCBI and protein data bank (PDB). Based on these data's, the comparative model of monoclonal antibody onto the viral surface antigen that can be predicted. We performed selected 500 antibodies sequence retrieved from the NCBI and PDB server to validate and filtered by molecular dynamics (MD) simulation (Wallraven et al., 2020) according to the best correlation between calculated and measured binding affinities in between the target of nCoV-2 Spike-S1 RBD epitopes specific antigen to monoclonal antibody. 


\section{Identification of antigen and antibody sequence}

A vaccine trains the body's immune system to recognize some viral antigen signatures that were present in Spike-S1 protein specific antigen in the RBD. The nCoV-2 is a recombinant antigen, which contains normal amino acids $408-470$ and 540-573 of the Spike-S1 protein immunodominant region. Since we identified specific antigenic epitopes of small synthetic peptide are regions of protein surface in RBD to the target of antibody by two different programs the immune epitope database (IEDB) (Jespersen et al., 2017) and Support Vector Machine (SVM) Tri-peptide similarity and Propensity (SVMTriP) (Yao et al., 2012). We identified consists of antibody sequence in two chains (VL/VH) after filtered by MD simulation in out of 500 from NCBI and PDB. In chain A-VL (light chain): Cl4mAb synthetic construct with GenBank ID: AEW26702.1. In chain B-VH (heavy chain): Cl4mAb synthetic construct with GenBank ID:

AEW26701.1.

\section{Structural design of antigen and antibody}

Antibody structure was determined and validate by two novel programs. First the structural antibody database (SAbPred) based on prediction relay on sequence homology to experimentally determined structures (Matthew et al., 2019 and James et al., 2016). It can be used to produce models of the antibody Fv region, or nanobodies. Number the sequences of both heavy and light chain aligned by Antigen receptor Numbering And Receptor Classificatlon (ANARCI) (James et al., 2016). Finally choose the best templates of $\mathrm{VH}$ and $\mathrm{VL}$ domains separately using SAbDab. Predict $\mathrm{VH}-\mathrm{VL}$ orientation by using $A B a n g l e$ and place the template domains in the predicted pose. A selected model of complementaritydetermining regions (CDR) loops with FREAD, it has been shown to produce very accurate results, regardless of loop length. In particularly antibody side chain predict with position dependent antibody rotamer swapper (PEARS) (James et al., 2016), and further confirmation of renumber the final models with ANARCI. End card of the structural annotate were standardized by following category of estimated model accuracy, recognizes potential develop ability issues and visualize different regions of the structural antibody. Secondly ABpredict2 determined program for antibody prediction (Gideon et al., 2019). Models are not separated to light and heavy chains and are numbered according to Chothia numbering for the prediction by using sequence homology, conducts a Monte Carlo-based search for lowenergy combinations of backbone conformations to yield accurate and unstrained antibody structures. The RMSD estimates above $90 \%$ sequence identity were calculated by using the entire set of full dataset sequences as well using TM-align did calculation. Further targeted antigen structure carried out by PDB ID: 6VW1 structure of 2019-nCoV-2 receptor-binding domain (RBD) complexed with its receptor human (ACE2).

\section{Protein-to-protein interactions and structures validation}


To conduct initial sampling of antigen and monoclonal antibody was used by the protein-to-protein docking server ClusPro 2.0 (Vajda et al., 2017), which is based on a docking program called PIPER2 (Kozakov et al., 2006). Which was more effective docking algorithm for antibody-protein antigen complex prediction is an important first step toward design of biologics and vaccines. PIPER is an FFTbased docking program that uses a structure-based pairwise potential as one component of its energy function. The total energy was the sum of terms representing shape complementarity, electrostatic and desolvation contributions by the pairwise potential (Chuang et al., 2008). For result assessment, we consider the model a hit if ligand of antigen atoms within $10 \mathrm{~A}^{\circ}$ of the receptor of monoclonal antibody are within $10 A^{\circ}$ RMSD in the model to ignore the motion of that part of the ligand which is not participating in the interaction. To evaluate our docking of the receptor and ligand potentials was incorporated as the pairwise component of the energy function used in PIPER, followed by clustering of the top 1000 results for evaluate (Kozakov et al., 2006), as implemented in our protein-protein docking server ClusPro (Comeau et al., 2004) that was here in more advantage.

\section{Prediction of protein-protein interaction interfaces hot spots}

The amino acid residues in the binding region of proteins is not uniform rather, they contain critical residues, called hot spot. Hot spots are primary targets of therapeutic agents, because designing a molecule that will bind to hot spots may lead to the disruption of a protein-protein interaction. We predict hot spots for our both antigen and antibody used by PRISM Concepts (Baspinar et al., 2014 and Tuncbag et al., 2011). In experimentally, hot spots are determined by alanine scanning mutagenesis where if the contribution of the mutated residue to the binding is more than $2.0 \mathrm{kcal} / \mathrm{mol}$ these residues are labeled as hot spots. If the distance of any two atoms between residues was less than their sum of van der Waals radii plus 0.5 Angstrom, both residues were registered as interfaced residues. When an assigned interface residue was less than a 10 residues, an arbitrary but reasonable number to reflect the minimum requirement of contact, the interface was considered as a result of crystal packing force.

\section{Results}

\section{Antibody structural annotation of CDR's for RBD and estimated accuracy}

Our targeted antibody sequence are Chothia-numbered by ANARCI. The numbered sequences was Chothia- aligned with more-than 2,000 high quality antibodies with known structure from SAbDab. For each dataset sequence from an Ig-seq, the alignment identifies the best structural templates for the full variable region and framework independently. FREAD then identifies, if any, the most suitable template structures for the CDRs. CDRs are germline to obtain a more comprehensive structural interpretation of the Ig-seq CDRs for which FREAD do not find direct matches to the PDB, but predicted the loop conformation from the targeted of our antibody sequence only. Well over $50 \%$ of the unique sequences of non-H3 CDRs can be accurately structurally predicted. This appears to recapitulate on a large scale the 
canonical shape phenomenon of non- H3 CDRs. In the case of H3, even though there were only a handful of direct PDB matches $\mathrm{H} 3$ sequences, according to FREAD estimates to produce structural models for 65 $\%$ of all non-redundant and $75 \%$ of redundant H3 loops (Table 1 ).

The antibody structure that the specific physicochemical configuration, which modulates the molecule's specificity and affinity. Binding shape biases in antibody repertoires was insight into the strategies of the immune system for tackling arbitrary RBD antigen. For instance, sequence similarities can be indicative of shared RBD antigen specificity. CDRs identified the physicochemical properties of the paratope, identifying potential RBD antigen-specific of our antibody. Such structural annotations were accurate as more paired Ig-seq datasets as based on these data allow the entire Fv regions as followed the full frameworks $\mathrm{VH}$ : $(\mathrm{H} 1, \mathrm{H} 2, \mathrm{H} 3)$ and $\mathrm{VL}(\mathrm{H} 1, \mathrm{H} 2, \mathrm{H} 3)$ was modeled rather than separate heavy and light chains (Figure 2). Our monoclonal antibody for targeted RBD of specific antigenic epitope annotations was employed in immunodiagnostics to find antibody-markers of known viral diseases. Therefore, employing structural information provides novel ways to study the diversity of the immune system. The estimated accuracy of modelled VH and VL domains procedure has been benchmarked. Result was estimated each region of the model was likely to have been predicted $75 \%$ of $\mathrm{VH}$ framework structures with sequence identity of $80 \%+/-2.5 \%$ have a backbone RMSD of $1 \AA ̊$ or better. Therefore the model has been predicted based on our antibody sequence with $80 \%$ sequence identity to the target we have a confidence of $75 \%$ that the VH framework was modelled with $1 \AA$ RMSD.

Antibody designed by another program AbPredict for binding affinity in between $\mathrm{VH} / \mathrm{VL}$ comparison. For this modeling same consists with a random combination of four backbone fragments a two fragments for the $\mathrm{VL}$ and $\mathrm{VH}$, which comprise CDRs 1 and 2 and the light and heavy chain framework regions, respectively, and two fragments for LCDR3 and HCDR3, it then our antibody sequence on the backbone fragments and performs a simulated annealing Monte-Carlo search over all conformational degrees of freedom. In each conformational move, the method randomly samples from pre-computed databases a backbone conformation belonging to the VL, VH, LCDR3, HCDR3 followed by combinatorial side-chain packing and side-chain and backbone minimization to reduce stereochemical strain. At the end of each trajectory, the lowest-energy structure sampled during the trajectory. The result of antibody Fv region accuracy and stereochemical strain in models produced for eight antibodies that were part of the AMA-II blind benchmark of monoclonal antibody structure modeling (Figure3). Both programs estimated that were mostly given best antibody deviated by $<1.2 \AA$ rmsd over backbone-carbonyl atoms and exhibited stereochemical quality that is expected of structures at resolutions $<1.2 \AA$. The 500 resulting models were clustered by carbonyl rmsd and the finally lowest-energy structures from the top-three clusters.

\section{Antigenic epitope of nCoV2-Spike-S2-RBD binding interaction with monoclonal antibody}

The nCoV-2 assortment was reflected in the variable spike proteins (S proteins), which have evolved into forms differing in their receptor interactions and their response to various environmental triggers of virus- 
cell membrane fusion. It was mainly infect the human respiratory epithelial cells through interaction with the hACE2 receptor. Indeed, the recombinant Spike protein can bind with recombinant hACE2 protein. The S1 contains two subdomains, a N-terminal domain (NTD) and a C-terminal domain (CTD) both are able to function as receptor binding domains (RBDs) and bind variety of proteins and sugars. The S2 domain was typical of a class I viral fusion proteins. Heptad repeats comprise a repetitive heptapeptide with being hydrophobic residues characteristic of the formation of coiled-coil that participate in the fusion process. The nCoV-S1 complexed with human hACE2 provided the fissionable view of coronavirus $\mathrm{S}$ (Figure 4), which contains two subdomains as core structure and RBD. A five-stranded anti-parallel $\beta$ sheet $(\beta 1-\beta 4$ and $\beta 7)$ that connects with three short $\alpha$-helices $(\alpha A-\alpha C)$ constitutes the core, whereas a two-stranded $\beta$-sheet ( $\beta 5$ and $\beta 6$ ) forms the loop. $N^{*}$ and $C^{*}$ represent the amino and carboxyl termini of RBD. The RBD gently concave outer surface to bind hACE2. The base of this concave surface was a short, two-stranded antiparallel $\beta$-sheet, and two ridges are formed by loops. The ectodomain of hACE2 contains a membrane-distal peptidase domain and a membrane-proximal collecting domain. The RBD of $S$ protein contains multiple conformation-dependent with different epitopes and the main domain that induces neutralizing antibody. We identified entire regions antigenic epitope of small peptide even single amino acid in the RBD that was taken place from predict liner antigenic epitopes (SVMTrip). It was consists of four selected epitopes region were in [DDVRQIAPGQTGVI], [NIDATSTGNYN], [YQAGSTPCNGV], and [YGFQPTNGVGYQ], are mainly antigenic properties which are directly interact with the monoclonal antibody (Table 2). A calculation of a high rate of the binding affinity between RBD and antibody was $-12.1 \mathrm{kcal} / \mathrm{mol}$.

We identified two amino acids Asn479 and Thr487 of the RBD are important for the interaction highaffinity overtone of S protein with hACE2. Particular mutations at Arg441, and Asp454 of the RBD disrupt the antigenic structure and binding activity of RBD to hACE2. Accordingly boosted our antibody stability and affinity in to Fv framework regions (CDRs) which are typically in direct contact with the antigenic epitope for instance, improved electrostatic complementarity with the antigen of RBD affinity of monoclonal antibody by which altered amino acids in hydrophobic surface patch. There are four epitopes regions of the RDB were identified but since only three are the best epitopes by rank were interacts with the monoclonal antibody (Figure 5). This monoclonal antibody were interacting regions of RBD in out of three the best-selected rank one that are main of our target. This same region of RBD binding with the hACE2 receptor and neutralize infection. Monoclonal antibody of $\mathrm{VH}$ chain 1 were interact antigenic epitope binding hotspot residues of the RBD (Pro38-Val505, Tyr58-Asn437, Phe109-Val503, Phe109Gly502, Phe110- Gly502, Phe110-Tyr505, Phe109-Tyr505, Tyr64-Arg439, Tyr64-Asn440, Ser57-Arg439, Phe109-Gly504,

Asn113-Thr500, Tyr66-Arg439, Asn113-Gly502 and Tyr66-Pro499) as for VL chain 2 were interact antigenic epitope binding hotspot residues of the RBD (Glu109-GIn498, Glu109-Thr500, Asn108-Thr500 and Trp114- Thr500) (Table 3). In due to the interactions direct H-bonds across the epitope to paratope interfaces could contribute to the specificity of the antibody to antigen recognition that was short-chain hydrophilic side chains are particularly suitable for this interaction because of the smaller side-chain conformational entropy penalty in the interface. 


\section{Optimizing Lead antibody of affinity, specificity, and stability}

The mutated $\mathrm{VH}$ and $\mathrm{VL}$ domain combinations by developed a new antibody ( $>108$ additional diversity) with predefined antigen specificity. Compared to the hit discovery, it can be used for in-vitro selection under more stringent conditions to identify antibodies with improved characteristics. The most important antibody properties relate to their natural functions, such as they're high binding affinity and specificity mediated by their complementarity determining regions of monoclonal antibody (CDRs) within the variable VL/VH chains. Other key natural antibody properties include their effector functions in both dependent cell mediated of complement and dependent cytotoxicity. We created designed library of variants of RBD-monoclonal antibody interface. These structures contain a complex of two proteins, one in "RBD: Chain F" and the other in $\mathrm{VH}$ : "Chain $\mathrm{H}$ " and "Chain L". The proteins interact through an interface comprises a conserved core, known as the interaction hotspot (Figure 6). Conformations of the side chain hotspot regions by simulations interaction from protein complex in between monoclonal antibody and $\mathrm{RBD}$, which order to increase its affinity for the target chain of RBD. The result of proteins interact through an interface formed by three loops amino acid position of regions were in 473-483: YQAGSTPCNGV, 441451: NIDATSTGNYN and 495-506: YGFQPTNGVGYQ on chain F (RBD) which interact with monoclonal antibody. Embracing of these two regions amino acid regions 473-483: YQAGSTPCNGV and 441-451: NIDATSTGNYN, which encodes much of the binding affinity, and peripheral interactions, where binding incompatibility toward other natural encoded.

Additionally, the periphery of the binding interface, make important contributions to specificity. Inspired by this modularity of binding interfaces, while conserving the interaction hotspot and optimizing the rigidbody orientation and sequence of other interfacial regions for further conformation periphery of the binding interface, the main implicit that backbone designs that retained the structural geometry would allow the remainder of the our complex proteins, including the essential hotspot, to fold to the native conformation, thus maintaining high-affinity binding in between monoclonal antibody and RBD.

The association between the light and heavy domains in immunoglobulins, and these can be specifically associated with key residues in our monoclonal antibody sequence. Belong the sequence of CDRs rest on a conserved framework, which provides the biochemical properties of structural stability. Structural diversity in the Fv framework region was experimental primarily in the CDRs, which vary in length, backbone conformation, and amino acid sequence. The rigid-body orientation of the light relative to the heavy chain was another important that affects the conformation of the RBD antigen-binding site. Furthermore confirmation of protein stability effect of salts dependent and it was a complex balance of the multiple mechanisms by which the ionic salt interacts with protein molecules, shielding charged solvent exposed residues and then potentially decreasing protein-protein long-range electrostatic interactions.

\section{Discussion}


The Spike's receptor binding domain is the primary determinant of a coronavirus's ability to infect a host species and its tropisms. SARS-CoV-2's genome shows that its closest human-infecting relative is the Severe Acute Respiratory Syndrome Coronavirus (SARS-CoV), with a sequence identity of approximately $79 \%$ (Roujian et al., 2020). Identity between the spike proteins is $76.47 \%$, with slightly lower sequence identity between the RBDs (73 \%) (Xu et al., 2020). Within SARS-CoV-2's 193 residues RBD, a large proportion of mutations are highly accumulated at the C-terminal region that includes the receptorbinding motif (Yushun et al., 2020). The RBD in S1 is responsible for virus binding to host cell receptors (Wong et al., 2004, Li 2003). Neutralizing antibodies and T-cell immune responses can be raised directly against several nCoV-2 proteins (S, E, M and N) (Wang 2020, Zhou 2018, Du2016, See 2008), but mainly target the $S$ protein (Zakhartchouk et al., 2007), suggesting that to induced specific immune responses on the surface of the viral particle plays key roles in the binding of the cell receptor and membrane fusion (Cui et al., 2029). The hACE2 is expressed and active in various cell types including alveolar epithelial cells, surface enterocytes of the small intestine, and heart and kidney endothelial cells (Sims et al., 2005). Essentially the $S$ protein of $n \mathrm{CoV}-2$ is involved in the hACE2 receptor recognition, as well as virus attachment and entry it represents one of the most important targets for the development of nCoV-2 vaccines and therapeutics.

In the study, we choose the spike protein, as a candidate target to monoclonal antibody way to stop the virus infection is to block the RBD with hACE2 interaction. We designed antibody in each conformational passage, the method randomly samples from pre computed dataset a backbone conformation belonging to the antibody of VL, VH, L-CDR3, H-CDR3 in Fv framework by combinatorial Sidechain packing and Sidechain and backbone minimization to reduce stereochemical strain (Lapidoth et al., 2015). Based on we analyzed the accuracy and stereochemical strain in models produced for eight antibodies that were part of the AMA-II blind benchmark of antibody structure modeling methods (Almagro et al., 2014). Our top models exhibited stereochemical quality that was expected of structures at resolutions $<1.2 \AA$ rmsd. The 500 resulting antibody models are clustered by carbonyl rmsd and the lowest-energy structures from the top-three clusters.

Our monoclonal antibody was highly interacting binding with a specific antigenic epitope to the RBD. During the interaction of RBD with the receptor, RBD presents a concave surface for the $\mathrm{N}$ terminus of the receptor peptidase, on which amino acids 441-451: NIDATSTGNYN broadcasters the entire receptor binding loop of RBD core. These loop amino acids 473-483: YQAGSTPCNGV of the RBD, which makes directly complete contact with the antibody. The RBD region also contains multiple cysteine residues that are linked by di-sulphide bonds ( $\mathrm{Li}$ et al., 2005). These two amino acid regions in particular, those at positions 441-451 and 473-483, determine nCoV-2 disease progression (Li et al., 2005). Any residues changes in these two positions might therefore develop animal-to-human and human-to-human transmission (Arcilla et al., 2017). Further to the structure of RBD, residue $498 \mathrm{GLN}$ in nCoV-2 was located very near virus-binding hot spot $500 \mathrm{Thr}$ (i.e., hot spot 109Glu and $110 \mathrm{Phe}$ ) on antibody. Hot spot 353 consists of a salt bridge between Lys353 and Asp38 also buried in a hydrophobic environment (Wu et al., 2012). 
The RBD are important for the high-affinity association of S protein with hACE2 (Li et al., 2005). A point mutation at Arg441, Asp454 of the RBD disrupts the antigenic structure and binding activity of RBD to hACE2 (He et al., 2006). Our antibody structure improved interactions across the VL-VH interface result in substantial optimization of a range of essential parameters for antibody development, including impressibility, stability, and affinity which more highly capable interact with antigenic epitope to the RBD which mainly antibody core alter according to the structure of antigen-binding site, these interactions contribute the majority of the binding energy for the antibody to protein complexes as hot spots in the interfaces. The designed mutations cooperate with surface mutations identified through conventional antibody to increased affinity and stability. Finally, taken together, this study provides developed monoclonal antibody model and together of essential function for the development of nCoV-2 RBD for therapeutics to completely prevent vial infection.

\section{Future Directions:}

Overall we successfully designed new monoclonal antibody for novel coronavirus based on $\mathrm{S}$ protein to the targeted of specific antigenic epitope to the RBD to inhibit the host viral entry. Future studies to understand immunologic factors and antibody pathways associated with the successful development of antibodies may provide insights for the design of improved immunogens and immunization strategies.

\section{Declarations}

\section{Acknowledgement:}

I thanks the World Health Organization (WHO) continue to update the Covid19 platform with new data sets from national and sub-national level, as well as showing data of clinical trials, country-specific public health measures, and other important indicators which is highly supported to my research work. We collaborate our research analysis was partially performed at the Center for High-Performance Computing, the regional research laboratory, Ministry of Health, Makkah, The Kingdom of Saudi Arabia.

\section{Disclaimer:}

The study sponsor had no role in study design, data collection, data analysis, data interpretation, or writing of the report. The first and corresponding author's had full access to all study data and had final responsibility for the decision to submit for publication.

\section{Financial support:}

The Deanship of Scientific Research, Science and Technology Unit, and Faculty of Medicine at Umm AlQura University (UQU) of the Kingdom of Saudi Arabia to Dr. Zainularifeen Abduljaleel funded this work. 


\section{Potential conflicts of interest:}

All authors: No reported conflicts of interest. All authors have submitted form for Disclosure of Potential Conflicts of Interest. Conflicts that the editors consider relevant to the content of the manuscript have been disclosed.

\section{Author contributions:}

Z.A., and F.A. designed research contributed new reagents. Analytic tools data analysis; Z.A. Analyzed data; F.A., and Z.A. wrote the paper. Principle Investigator; Z.A.

\section{References}

1. Almagro, J.C. et al. Second antibody modeling assessment (AMA-II). Proteins 82, 1553-62 (2014).

2. Arcilla, M.S. et al. Import and spread of extended-spectrum $\beta$-lactamase-producing Enterobacteriaceae by international travellers (COMBAT study): a prospective, multicentre cohort study. Lancet Infect Dis 17, 78- 85 (2017).

3. Baspinar, A., Cukuroglu, E., Nussinov, R., Keskin, O. \& Gursoy, A. PRISM: a web server and repository for prediction of protein-protein interactions and modeling their 3D complexes. Nucleic Acids Res 42, W285-9 (2014).

4. Cui, J., Li, F. \& Shi, Z.L. Origin and evolution of pathogenic coronaviruses. Nat Rev Microbiol 17, 181$192(2019)$.

5. Du, L., Tai, W., Zhou, Y. \& Jiang, S. Vaccines for the prevention against the threat of MERS-CoV. Expert Rev Vaccines 15, 1123-34 (2016).

6. Dunbar, J. \& Deane, C.M. ANARCI: antigen receptor numbering and receptor classification. Bioinformatics 32, 298-300 (2016).

7. Dunbar, J. et al. SAbPred: a structure-based antibody prediction server. Nucleic Acids Res 44, W474-8 (2016).

8. He, Y., Li, J. \& Jiang, S. A single amino acid substitution (R441A) in the receptor-binding domain of SARS coronavirus spike protein disrupts the antigenic structure and binding activity. Biochem Biophys Res Commun 344, 106-13 (2006).

9. Jespersen, M.C., Peters, B., Nielsen, M. \& Marcatili, P. BepiPred-2.0: improving sequence-based B-cell epitope prediction using conformational epitopes. Nucleic Acids Res 45, W24-W29 (2017). 
10. Kozakov, D., Brenke, R., Comeau, S.R. \& Vajda, S. PIPER: an FFT-based protein docking program with pairwise potentials. Proteins 65, 392-406 (2006).

11. Lapidoth, G.D. et al. AbDesign: An algorithm for combinatorial backbone design guided by natural conformations and sequences. Proteins 83, 1385-406 (2015).

12. Lapidoth, G., Parker, J., Prilusky, J. \& Fleishman, S.J. AbPredict 2: a server for accurate and unstrained structure prediction of antibody variable domains. Bioinformatics 35, 1591-1593 (2019).

13. Li, F., Li, W., Farzan, M. \& Harrison, S.C. Structure of SARS coronavirus spike receptor-binding domain complexed with receptor. Science 309, 1864-8 (2005).

14. Li, W. et al. Angiotensin-converting enzyme 2 is a functional receptor for the SARS coronavirus. Nature 426, 450-4 (2003).

15. Li, W. et al. Receptor and viral determinants of SARS-coronavirus adaptation to human ACE2. EMBO J 24, 1634-43 (2005).

16. Li, Z. et al. Development and clinical application of a rapid IgM-IgG combined antibody test for SARSCoV- 2 infection diagnosis. J Med Virol (2020).

17. Lu, R. et al. Genomic characterisation and epidemiology of 2019 novel coronavirus: implications for virus origins and receptor binding. Lancet 395, 565-574 (2020).

18. Lin D, Liu L, Zhang M, Hu Y, et al. Evaluation of serological tests in the diagnosis of 2019 novel coronavirus (SARS-CoV-2) infections during the COVID-19 outbreak. Medxriv. (2020)

19. Ng, O.W. et al. Memory T cell responses targeting the SARS coronavirus persist up to 11 years postinfection. Vaccine 34, 2008-14 (2016).

20. Okba, N.M.A. et al. Severe Acute Respiratory Syndrome Coronavirus 2-Specific Antibody Responses in Coronavirus Disease 2019 Patients. Emerg Infect Dis 26(2020).

21. Raybould, M.I.J. et al. Five computational developability guidelines for therapeutic antibody profiling. Proc Natl Acad Sci U S A 116, 4025-4030 (2019).

22. See, R.H. et al. Severe acute respiratory syndrome vaccine efficacy in ferrets: whole killed virus and adenovirus-vectored vaccines. J Gen Virol 89, 2136-2146 (2008).

23. Sims, A.C. et al. Severe acute respiratory syndrome coronavirus infection of human ciliated airway epithelia: role of ciliated cells in viral spread in the conducting airways of the lungs. J Virol 79, 15511-24 (2005).

24. Tang, F. et al. IL-12 RB1 genetic variants contribute to human susceptibility to severe acute respiratory syndrome infection among Chinese. PLoS One 3, e2183 (2008). 
25. Tuncbag, N., Gursoy, A., Nussinov, R. \& Keskin, O. Predicting protein-protein interactions on a proteome scale by matching evolutionary and structural similarities at interfaces using PRISM. Nat Protoc 6, 1341-54 (2011).

26. Vajda, S. et al. New additions to the ClusPro server motivated by CAPRI. Proteins $85,435-444$ (2017).

27. Varghese, R., Mikyas, Y., Stewart, P.L. \& Ralston, R. Postentry neutralization of adenovirus type 5 by an antihexon antibody. J Virol 78, 12320-32 (2004).

28. Wallraven, K. et al. Adapting free energy perturbation simulations for large macrocyclic ligands: how to dissect contributions from direct binding and free ligand flexibility. Chem Sci 11, 2269-2276 (2020).

29. Wan, Y., Shang, J., Graham, R., Baric, R.S. \& Li, F. Receptor Recognition by the Novel Coronavirus from Wuhan: an Analysis Based on Decade-Long Structural Studies of SARS Coronavirus. J Virol 94(2020).

30. Wang, N., Shang, J., Jiang, S. \& Du, L. Subunit Vaccines Against Emerging Pathogenic Human Coronaviruses. Front Microbiol 11, 298 (2020).

31. Wong, S.K., Li, W., Moore, M.J., Choe, H. \& Farzan, M. A 193-amino acid fragment of the SARS coronavirus S protein efficiently binds angiotensin-converting enzyme 2. J Biol Chem 279, 3197-201 (2004).

32. Wu, F. et al. A new coronavirus associated with human respiratory disease in China. Nature 579, 265269 (2020).

33. Wu, K., Peng, G., Wilken, M., Geraghty, R.J. \& Li, F. Mechanisms of host receptor adaptation by severe acute respiratory syndrome coronavirus. J Biol Chem 287, 8904-11 (2012).

34. Xu, X. et al. Evolution of the novel coronavirus from the ongoing Wuhan outbreak and modeling of its spike protein for risk of human transmission. Sci China Life Sci 63, 457-460 (2020).

35. Xu, X. \& Gao, X. Immunological responses against SARS-coronavirus infection in humans. Cell Mol Immunol 1, 119-22 (2004).

36. Yao, B., Zhang, L., Liang, S. \& Zhang, C. SVMTriP: a method to predict antigenic epitopes using support vector machine to integrate tri-peptide similarity and propensity. PLoS One 7, e45152 (2012).

37. Zakhartchouk, A.N. et al. Immunogenicity of a receptor-binding domain of SARS coronavirus spike protein in mice: implications for a subunit vaccine. Vaccine 25, 136-43 (2007).

38. Zhao, J. et al. Antibody responses to SARS-CoV-2 in patients of novel coronavirus disease 2019. Clin Infect Dis (2020). 
39. Zhou, P. et al. A pneumonia outbreak associated with a new coronavirus of probable bat origin. Nature 579, 270-273 (2020).

40. Zhou, Y., Jiang, S. \& Du, L. Prospects for a MERS-CoV spike vaccine. Expert Rev Vaccines 17, 677-686 (2018).

\section{Tables}

Table1. Antibody Fv region of VL-VH domains

\begin{tabular}{|llll|}
\hline Fv Region & $\begin{array}{l}\text { Template PDB } \\
\text { (Chain) }\end{array}$ & Selection Method & Score \\
\hline VH framework & A & Sequence identity & 0.9 \\
\hline CDR H1 & A & CDR specific fread & 71 \\
\hline CDR H2 & A & CDR specific fread & 40 \\
\hline CDR H3 & A & CDR specific fread & 25 \\
\hline VL framework & B & Sequence identity & 0.97 \\
\hline CDR L1 & B & CDR specific fread & 65 \\
\hline CDR L2 & B & CDR specific fread & 43 \\
\hline CDR L3 & B & CDR specific fread & 47 \\
\hline VH-VL orientation & A\&B & Same as VH and VL & 0.94 \\
\hline
\end{tabular}

Table 2. Antigenic epitopes prediction to the RBD 


\begin{tabular}{|lllll|}
\hline Target Protein & Start & End & Antigenic Epitopes regions & Length \\
\hline Receptor binding domain (RBD) & 347 & 347 & F & 1 \\
\cline { 2 - 5 } & 402 & 402 & V & 1 \\
\hline 405 & 418 & DDVRQIAPGQTGVI & 14 \\
\hline 423 & 425 & YKL & 3 \\
\hline 441 & 451 & NIDATSTGNYN & 11 \\
\hline 461 & 163 & LKP & 3 \\
\hline 466 & 467 & RD & 2 \\
\hline 469 & 469 & S & 1 \\
\hline 473 & 483 & YQAGSTPCNGV & 11 \\
\hline 495 & 506 & YGFQPTNGVGYQ & 12 \\
\hline
\end{tabular}

Table 3. Interacting residues in between monocular antibody and RBD 
Interacting VL \& LH Chain resName_resNo Interaction Interacting RBD resName_resNo View

\begin{tabular}{|c|c|c|c|c|c|c|c|c|}
\hline $\begin{array}{l}\text { Protein } \\
\text { No. }\end{array}$ & Chain & $\begin{array}{l}\text { Amino } \\
\text { acid }\end{array}$ & Position & & $\begin{array}{l}\text { Protein } \\
\text { No. }\end{array}$ & Chain & $\begin{array}{l}\text { Amino } \\
\text { acid }\end{array}$ & Position \\
\hline \multirow[t]{19}{*}{ PDB1 } & $\mathrm{H}$ & PRO & 38 & $<->$ & \multirow[t]{19}{*}{ PDB2 } & $F$ & VAL & 503 \\
\hline & L & GLU & 109 & $<->$ & & $F$ & GLN & 498 \\
\hline & $\mathrm{H}$ & TYR & 58 & $<->$ & & $F$ & ASN & 437 \\
\hline & L & GLU & 109 & $<->$ & & $F$ & THR & 500 \\
\hline & $\mathrm{H}$ & PHE & 109 & $<->$ & & $\mathrm{F}$ & VAL & 503 \\
\hline & $\mathrm{H}$ & PHE & 109 & $<->$ & & $\mathrm{F}$ & GLY & 502 \\
\hline & $\mathrm{H}$ & PHE & 110 & $<->$ & & $\mathrm{F}$ & GLY & 502 \\
\hline & $\mathrm{H}$ & PHE & 110 & $<->$ & & $\mathrm{F}$ & TYR & 505 \\
\hline & $\mathrm{H}$ & PHE & 109 & $<->$ & & $\mathrm{F}$ & TYR & 505 \\
\hline & $\mathrm{H}$ & TYR & 64 & $<->$ & & $\mathrm{F}$ & ARG & 439 \\
\hline & L & ASN & 108 & $<->$ & & $\mathrm{F}$ & THR & 500 \\
\hline & L & TRP & 114 & $<->$ & & $\mathrm{F}$ & THR & 500 \\
\hline & $\mathrm{H}$ & TYR & 64 & $<->$ & & $F$ & ASN & 440 \\
\hline & $\mathrm{H}$ & SER & 57 & $<->$ & & $F$ & ARG & 439 \\
\hline & $\mathrm{H}$ & PHE & 109 & $<->$ & & $\mathrm{F}$ & GLY & 504 \\
\hline & $\mathrm{H}$ & ASN & 113 & $<->$ & & $\mathrm{F}$ & THR & 500 \\
\hline & $\mathrm{H}$ & TYR & 66 & $<->$ & & $\mathrm{F}$ & ARG & 439 \\
\hline & $\mathrm{H}$ & ASN & 113 & $<->$ & & $\mathrm{F}$ & GLY & 502 \\
\hline & $\mathrm{H}$ & TYR & 66 & $\leftrightarrow$ & & $\mathrm{F}$ & PRO & 499 \\
\hline
\end{tabular}

\section{Figures}




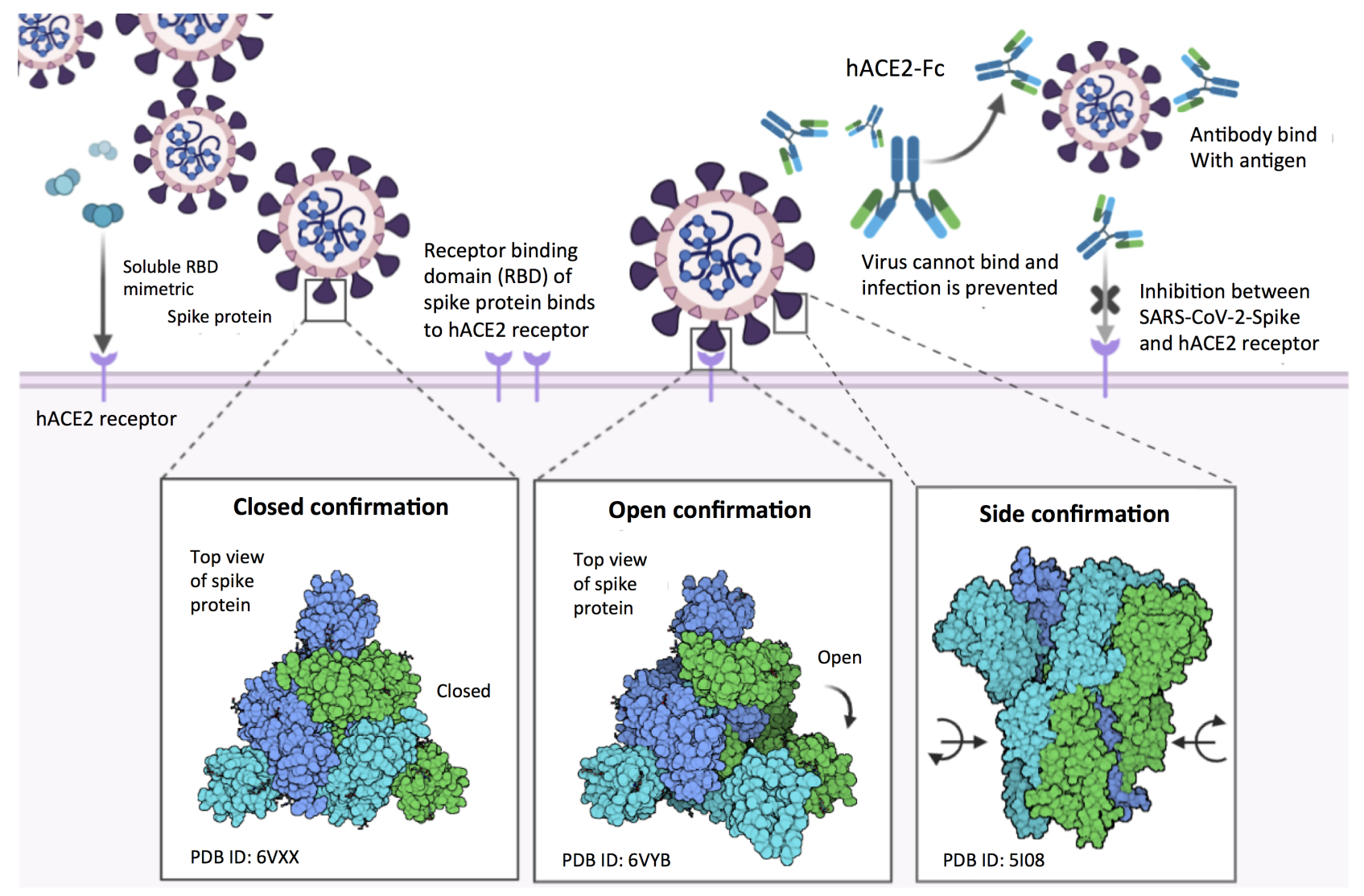

\section{Figure 1}

Tectonic conformational changes of SARS-nCoV-2: Spike-S protein. Human ACE2 is the host cell receptor responsible for mediating infection by SARS-nCoV-2, the novel coronavirus responsible for coronavirus disease 2019 (COVID-19). Cure with anti-hACE2 antibodies disrupts the interaction between virus and receptor. The Spike proteins on the surface of the coronavirus bind to angiotensin-converting enzyme 2 (hACE2) receptors on the surface of the target cell. In the process cleaved hACE2 and activated spike protein facilitate viral entry. In this function SARS-nCoV-2, the virus infection occurred in welldifferentiated ciliated epithelial cells expressing hACE2 thus the virus enters hosts through the mucosa of the respiratory and gastrointestinal tract. Once the viral membrane fuses with the human cell membrane, allowing the genome of the virus to enter human cells and begin infection. 


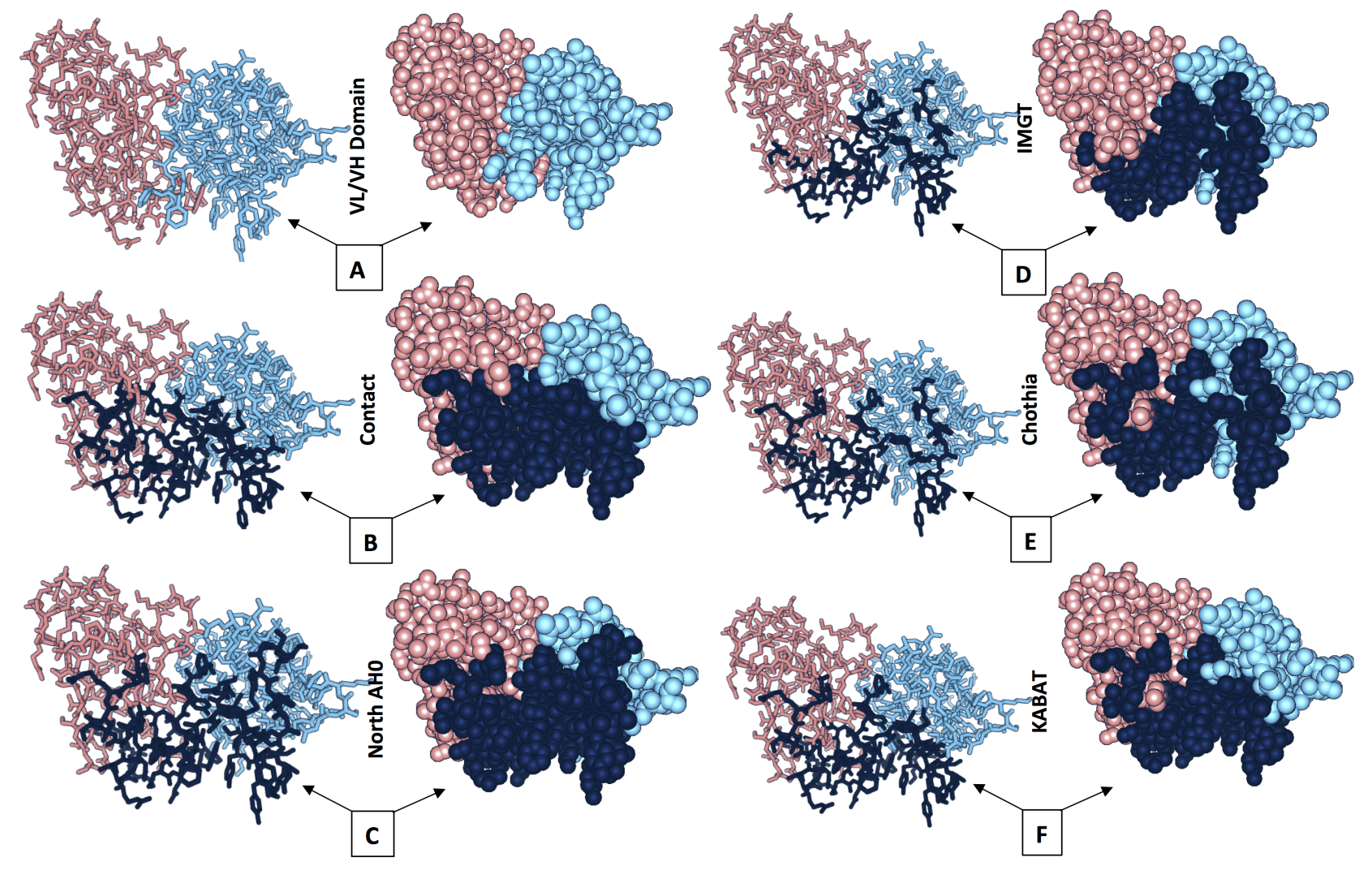

Figure 2

The antibody CDR's determined structural and classifications. (A) The antibody consisted of two domains VH/VL. (B) Highlighted of the antibody CDRs (VL/VH) residues color in blue most likely to be in contact with the RBD antigen. (C) North AHO are clustering of antibody CDRs (VL/VH) loop conformations residues highlighted in blue with high B-factors and high conformational energies. (D) IMGT are filtered to include of antibody variable domain sequences that are conserved residues color in blue CDRs (VL/VH) its derived from EMBL-ENA, (E \& F) Chothia and KABAT were annotated with key information such as CDRs (VL/VH) of potential post-translational modifications of residues to be highlighted color in blue and decisions to be made on which mutations to be acceptable. 

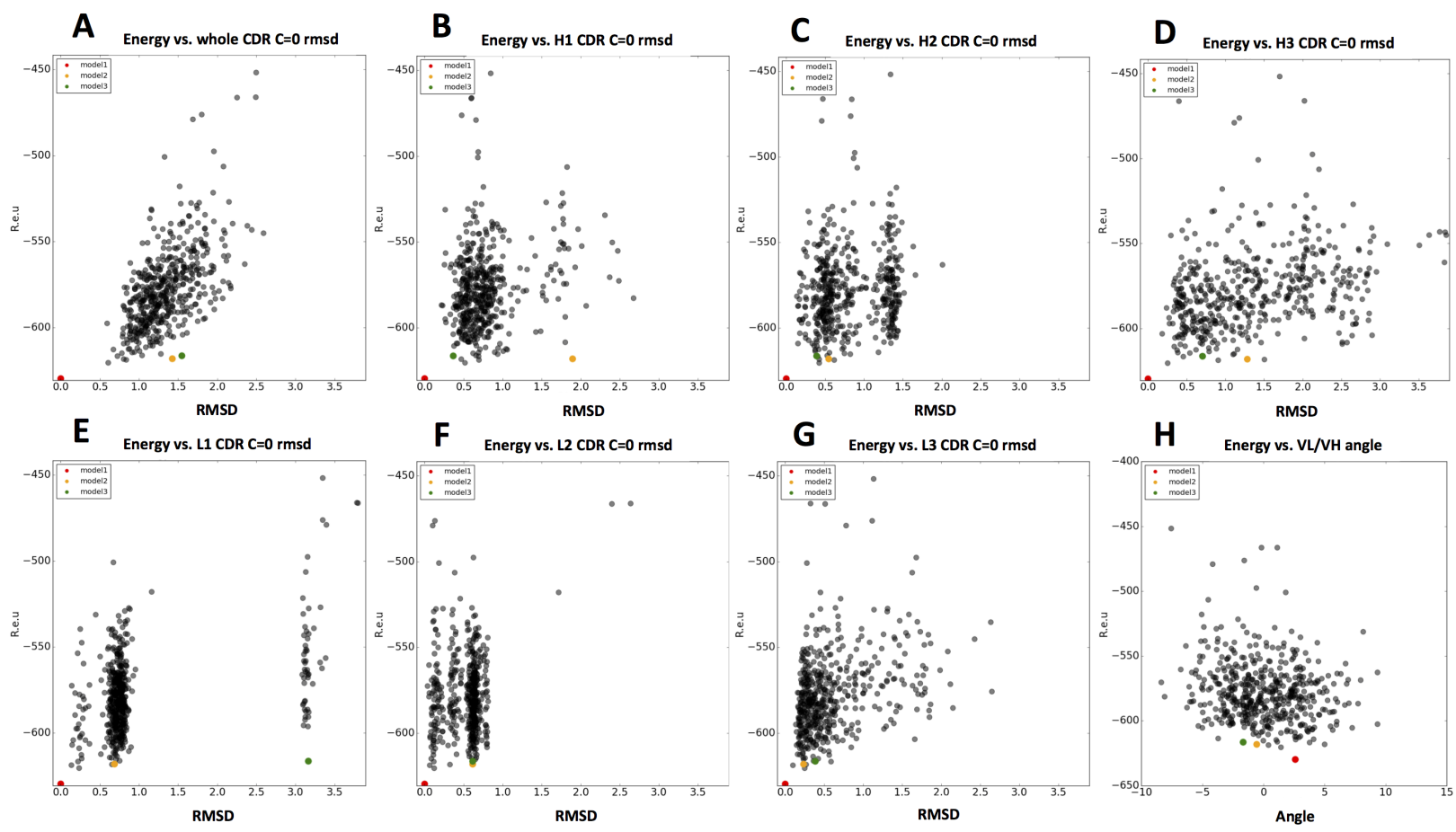

\section{Figure 3}

The antibody structure and altered energy minimization. The accuracy and stereochemical strain in models produced for eight query antibodies that were part of the antibody modeling assessment (AMA-II) blind benchmark of antibody structure modeling manners. A models exhibited stereochemical quality of structures at resolutions $<1.2 \AA$ by clustered by carbonyl rmsd and the lowest-energy structures resulting of top-eight models Fv regions were in $C D R C=0 H 1, H 2, H 3, C D R C=0 L 1, L 2, L 3$, and aligned with CDRs and antibody VL/VH model complex. 

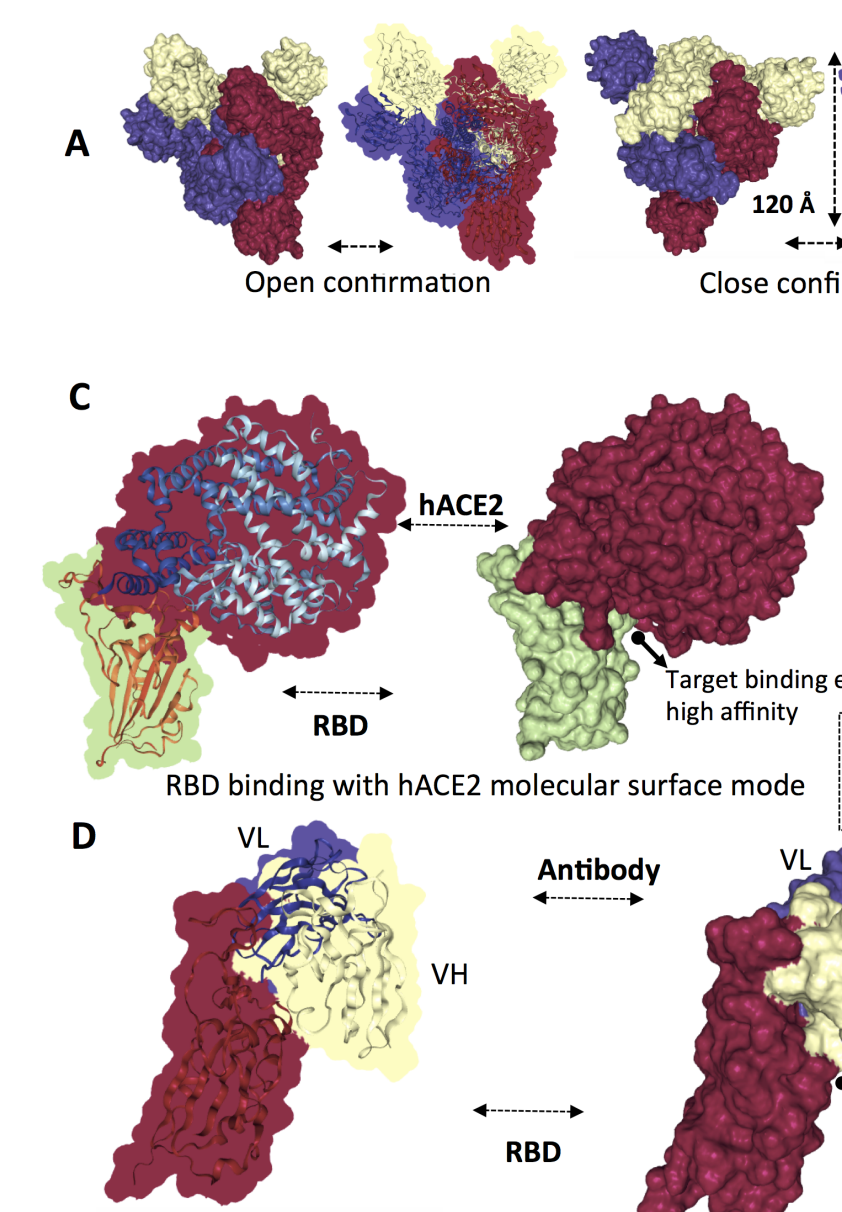

Close confirmation
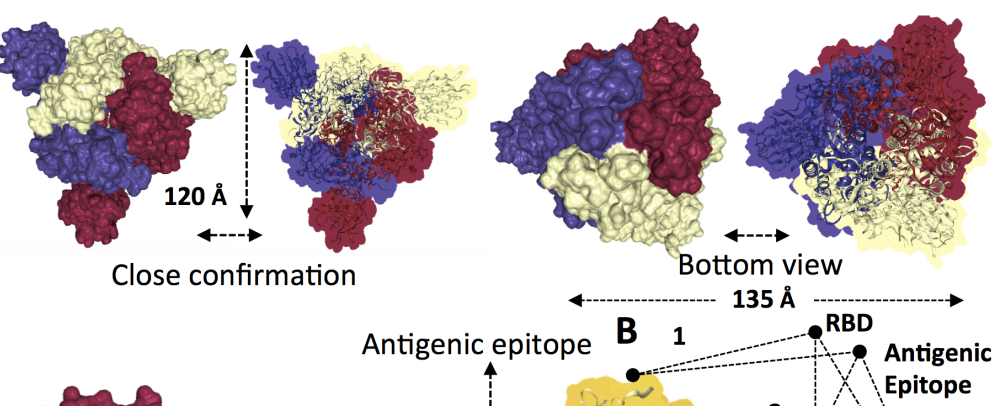

Antibody binding interact with epitope on an antigen of RBD

\section{Figure 4}

SARS-nCoV-2-Spike-S1 protein structure classification and antigen-antibody interface interactions. (A) The molecular surface and ribbon views of the structures are shown with different conformation i.e. (open and close view diameter $120 \AA$, top angle $135 \AA$ and side $140 \AA$ ). (B and C) The ectodomain of nCoV2 spike proteins are consists of two domains: a N-terminal domain named S1 that was responsible for receptor binding and a C-terminal S2 domain responsible for fusion. The spike S1 protein was a class I fusion protein a-helical coiled-coil structure was characteristic of this class of fusion protein, which contain in their C-terminal part regions predicted to have an a-helical secondary structure and to form coiled-coils with Horizontal angle $50 \AA$, and perpendicular angle. The S2 subunit was the most conserved region of the protein, whereas the $\mathrm{S} 1$ subunit diverges in sequence of a single coronavirus. The $\mathrm{S} 1$ contains two subdomains, a N-terminal domain (NTD) and a C-terminal domain (CTD). Both are able to function as receptor binding domains (RBDs) and bind variety of proteins and sugars. The $\mathrm{nCoV}$ diversity was reflected in the variable spike proteins (S proteins), which have evolved into forms differing in their receptor interactions and their response to various environmental triggers of virus to cell membrane fusion. It's been infect the human respiratory epithelial cells through interaction with the hACE2 receptor (red) and RBD (green). Indeed, the recombinant spike protein binds with recombinant hACE2 protein. (D) 
Antibody VL (blue) / VH (half white) binding interaction to the specific epitope with the RBD (red) which interact binding affinity was $-12.1 \mathrm{kcal} / \mathrm{mol}$.

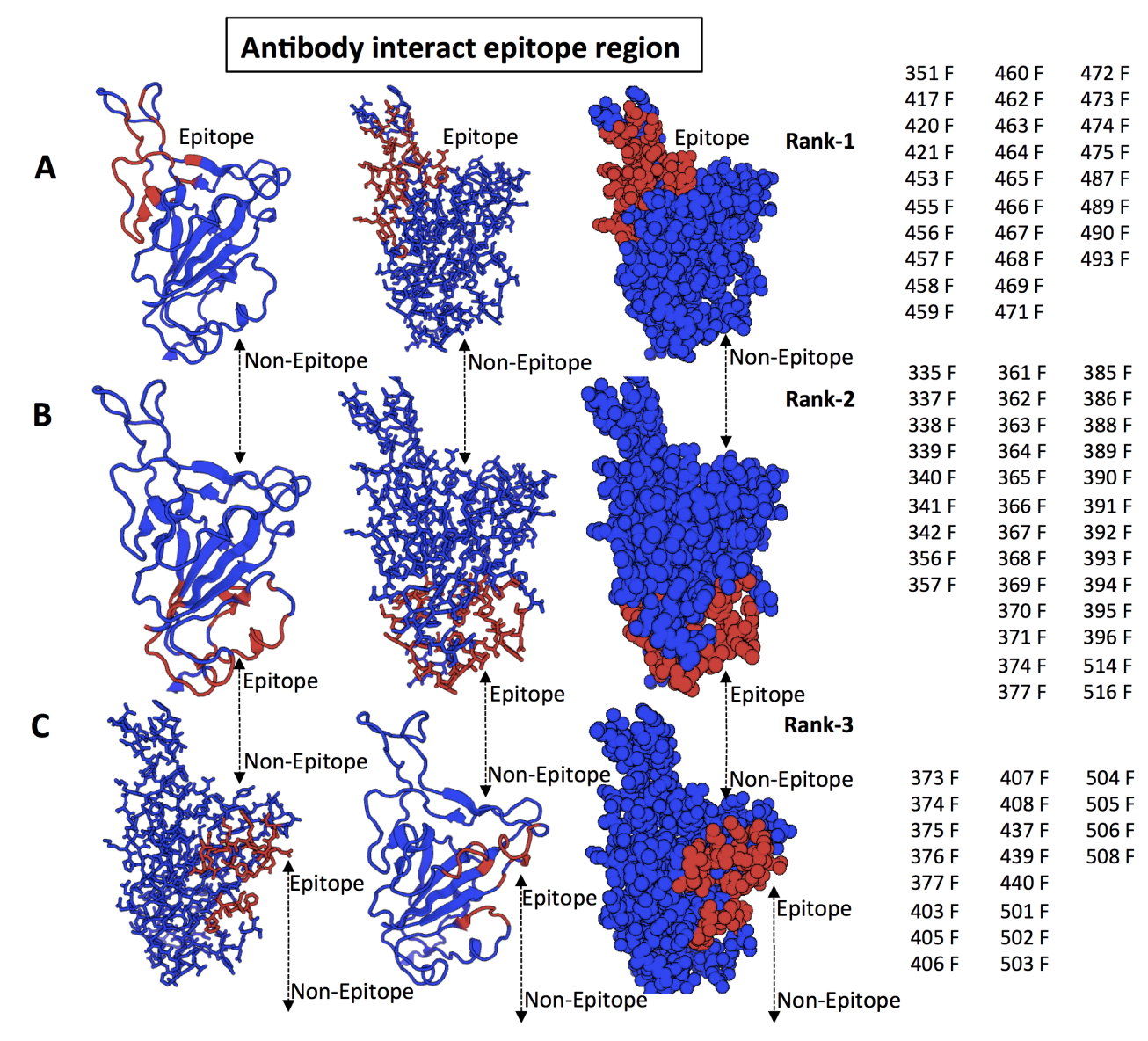

Figure 5

Interactions interface between antigenic epitopes and antibody binding site. Epitope was consecutive fragment from the amino acid sequence, and composed of several fragments scattered along the amino acid sequence, which form the antigenic regions of RBD-binding interface. Such predictions based on the amino acid properties were including hydrophobicity, solvent accessibility, secondary structure, flexibility, and antigenicity. (A). There are three antigenic epitopes regions was predicted based on rank1, which was highlighted color in red to a direct contact of RBD to the antibody with interacting high binding affinity other blue are non-epitope. (B) A rank2 antigenic epitopes was predicted regions are (red: epitope and blue: non-epitope) present at a lower of RBD. (C) A rank3 antigenic epitopes was predicted regions are (red: epitope and blue: non-epitope) which conserved at the forward-facing portion of the RBD to interact with antibody. (A-C) In all the segments of the RBD antigenic epitopes specific amino acids are listed a near the model of protein structure. 
A

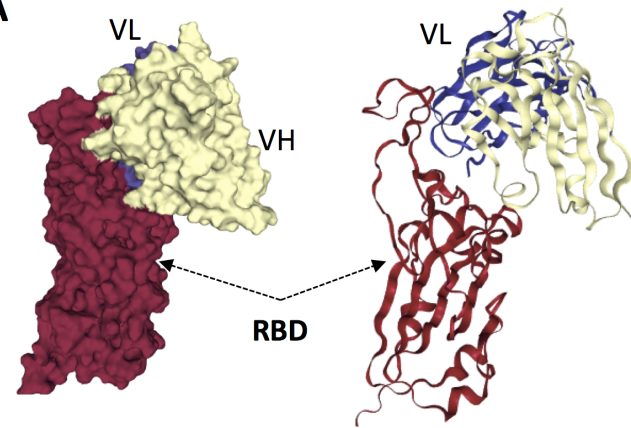

C Right angle: View confirmation

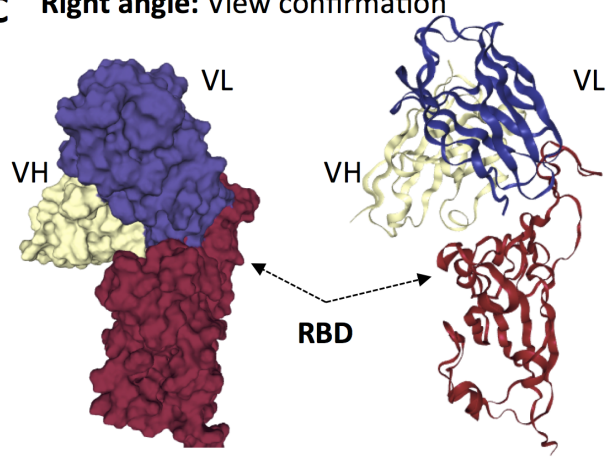

Front angle: View confirmation

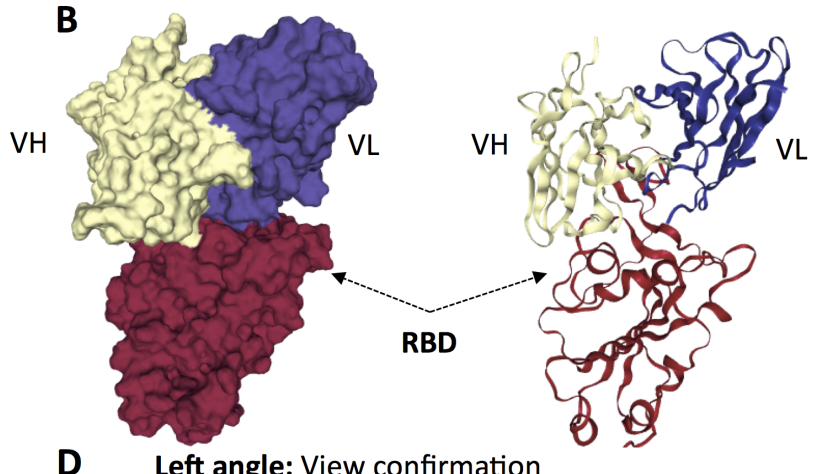

D Left angle: View confirmation

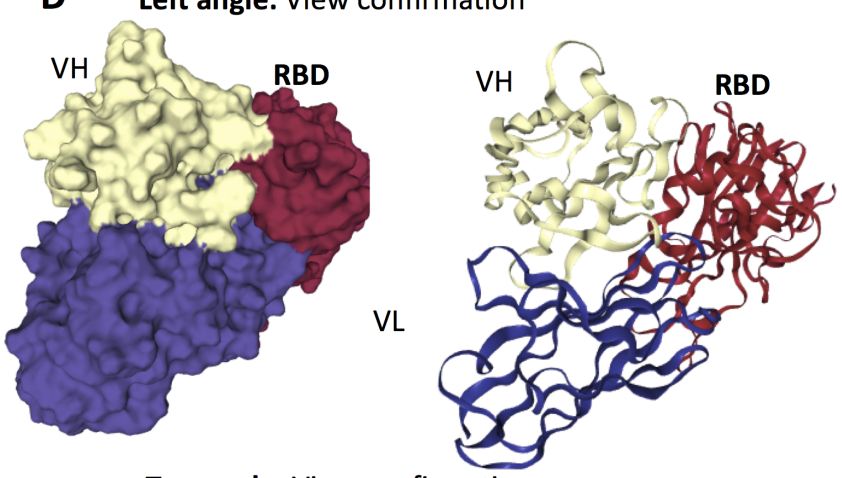

Top angle: View confirmation

\section{Figure 6}

SARS-nCoV-2 of RBD interactions with antibody This antibody that binds the RBD of SARS-CoV-2 challenged for the same antigenic epitope sites that bind the human receptor ACE2. (A-D) The antibody interacts with antigenic epitope of specific target bind to the RBD as shown by different orientations made on molecular surface and ribbon style (red: RBD, half white-VH/ blue-VL) 